\title{
Determination of Myosin Filament Orientations in Electron Micrographs of Muscle Cross Sections
}

\author{
Chunhong H. Yoon, Bjarni Bödvarsson, Søren Klim, Martin Mørkebjerg, Stig Mortensen, \\ James Chen, Student Member, IEEE, Julian R. Maclaren, Pradeep K. Luther, John M. Squire, \\ Philip J. Bones, Senior Member, IEEE, and R. P. Millane, Senior Member, IEEE
}

\begin{abstract}
An automated image analysis system for determining myosin filament azimuthal rotations, or orientations, in electron micrographs of muscle cross sections is described. The micrographs of thin sections intersect the myosin filaments which lie on a triangular lattice. The myosin filament profiles are variable and noisy, and the images exhibit a variable contrast and background. Filament positions are determined by filtering with a point spread function that incorporates the local symmetry of the lattice. Filament orientations are determined by correlation with a template that incorporates the salient filament characteristics, and the orientations are classified using a Gaussian mixture model. The precision of the technique is assessed by application to a variety of micrographs and comparison with manual classification of the orientations. The system provides a convenient, robust, and rapid means of analysing micrographs containing many filaments to study the distribution of filament orientations.
\end{abstract}

Index Terms-Disorder, electron micrograph, image analysis, lattice, muscle, myosin.

\section{INTRODUCTION}

$\mathbf{V}$ ERTEBRATE muscle fibers contain the contractile proteins myosin and actin which are organized into long thin strands called myofibrils [1]. The myofibrils are $2-5 \mu \mathrm{m}$ in di-

Manuscript received December 20, 2007; revised September 07, 2008. Current version published March 13, 2009. This work was supported in part by the N. Z. Marsden Fund, in part by the British Heart Foundation, and in part by the European MYORES network. The associate editor coordinating the review of this manuscript and approving it for publication was Dr. Birsen Yazici.

C. H. Yoon, P. J. Bones, and R. P. Millane are with the Department of Electrical and Computer Engineering, University of Canterbury, Christchurch, New Zealand (e-mail: chy26@student.canterbury.ac.nz; philip.bones@canterbury.ac.nz; rick.millane@canterbury.ac.nz).

B. Bödvarsson was with the Department of Electrical and Computer Engineering, University of Canterbury, Christchurch, New Zealand. He is now with SymCorp A/S, Copenhagen, Denmark (e-mail: b@bjarni.org)

S. Klim and S. Mortensen were with the Department of Electrical and Computer Engineering, University of Canterbury, Christchurch, New Zealand. They are now with the Department of Informatics and Mathematical Modelling, Technical University of Denmark, Lyngby, Denmark (e-mail: skli@novonordisk.com; sbm@imm.dtu.dk).

M. Mørkebjerg was with the Department of Electrical and Computer Engineering, University of Canterbury, Christchurch, New Zealand. He is now with Widex W/S, Vaerloese, Denmark (e-mail: mmb@widex.com).

J. Chen was with the Department of Electrical and Computer Engineering, University of Canterbury, Christchurch, New Zealand. He is now with the Department of Electrical Engineering, Stanford University, Stanford, CA 94305 USA (e-mail: james119@ cva.stanford.edu).

J. R. Maclaren was with the Department of Electrical and Computer Engineering, University of Canterbury, Christchurch, New Zealand. He is now with the Department of Diagnostic Radiology, University Hospital Freiburg, Freiburg, Germany (e-mail: julian.maclaren@uniklinik-freiburg.de).

P. K. Luther is with the National Heart and Lung Institute, Imperial College London, London SW7 2AZ, U.K. (e-mail: p.luther@imperial.ac.uk).

J. M. Squire is with the Department of Physiology and Pharmacology, University of Bristol, Bristol BS8 1TD, U.K. (e-mail: j.m.squire@bristol.ac.uk).

Color versions of one or more of the figures in this paper are available online at http://ieeexplore.ieee.org.

Digital Object Identifier 10.1109/TIP.2008.2011379 ameter and exhibit a periodic pattern along their length. The repeating unit is known as the sarcomere, is about $2.2 \mu \mathrm{m}$ long in resting vertebrate muscle, and is the basic contractile unit of muscle [1], [2]. The structure of the sarcomere is shown in Fig. 1 and consists of ordered transverse arrays of myosin and actin filaments. The myosin filaments lie on a triangular lattice with a spacing of $\sim 40 \mathrm{~nm}$, and the actin filaments lie on an interdigitated honeycomb lattice [Fig. 1(b) and (c)]. The myosin filament itself has a diameter of $\sim 15 \mathrm{~nm}$ [3]. Muscular force is generated by a series of biochemical reactions that result in a relative translation between the myosin and actin filaments. The translation is believed to result from a cycle in which myosin heads on the myosin filament surface attach to actin in the overlap region (Fig. 1), undergo a structural transition that produces relative movement, and then detach from actin [2], [4]. However, the exact molecular events that occur during this cycle are only partially understood and they are subject of intensive study in muscle structural biology. The myosin-actin interactions are a function of the geometrical arrangement of these molecules. Of particular importance is the rotational disposition of the myosin filaments within the myosin lattice [5], [6]. The geometry of the myosin lattice is, therefore, of fundamental importance in muscle biology.

X-ray fiber diffraction potentially provides high resolution information on whole muscle fibers, but the data are limited and their interpretation is difficult, particularly for systems that are only partially ordered, such as muscle [7], [8]. Electron microscopy, on the other hand, gives direct images of specially prepared muscle samples, with a resolution of $2-5 \mathrm{~nm}$ (depending on sample preparation), which is sufficient to resolve the individual myosin filaments as well as some of their characteristics [9]. The most clearly resolved images of the myosin lattice are obtained by taking carefully prepared thin sections through the so-called "bare-region" of the sarcomere (Fig. 1), where it is devoid of actin filaments and other molecular components, and imaging them in an electron microscope [3], [5]. Careful inspection of such images shows that the myosin filaments are approximately triangular in cross section and that the spatial distribution of their azimuthal rotations varies between muscle types and species [5], [6]. Henceforth, we will refer to the azimuthal rotation of a filament as its "orientation." In some muscles, such as those from teleost fish, the myosin filaments have the same orientation which is referred to as a "simple lattice" structure. However, in most vertebrate muscles, such as in tetrapods, the filaments adopt one of two different orientations that are spatially distributed in a semi-systematic manner within a single myofibril, referred to as a "superlattice" structure [5]. The two orientations differ by an azimuthal rotation of $60^{\circ}$ ( or $-60^{\circ}$ or 


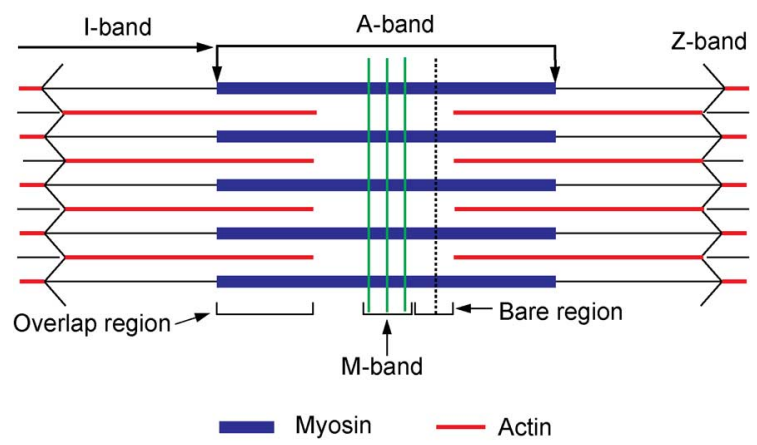

(a)

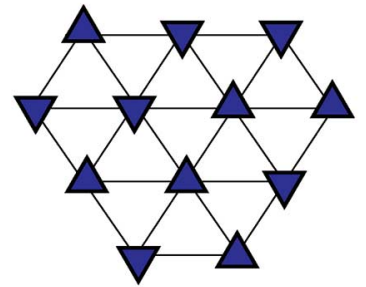

(b)

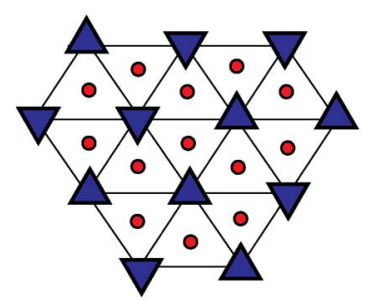

(c)
Fig. 1. Schematic of the muscle sarcomere structure. (a) Longitudinal section, (b) transverse section through the bare region showing the myosin filaments (represented by triangles) located on a triangular lattice, and (c) transverse section through the overlap region showing the myosin and actin (represented by circles) filaments.

$180^{\circ}$ as a result of their triangular shape). Quantitative information on the spatial distribution of the filament orientations is important for muscle structural biology since it has implications for the nature of the myosin-actin interactions and other aspects of muscle contraction. Furthermore, a statistical description of the distribution of the orientations is needed for rigorous interpretation of X-ray diffraction data from muscle fibers. Manual analysis of electron micrographs has allowed some general characteristics of the distribution of orientations to be determined [5], [10]. However, the noise level in the micrographs can be quite high and there can be as many as 1000 filaments to locate in a single myofibril cross section. Manual analysis, therefore, has problems in terms of operator fatigue and reproducibility of results. In this paper we describe an automated method for fast and accurate determination of the myosin filament orientations in electron micrographs of cross sections of the bare region of vertebrate muscle. Preliminary results for some parts of the algorithm have been reported previously [11]-[13].

A single micrograph usually intersects a number of myofibrils and a part of a micrograph that shows a single myofibril is shown in Fig. 2. The myofibril is the ordered region that is bounded by amorphous material, and the myosin filaments are the dark regions that are seen to lie on an approximately regular triangular lattice. There are large variations between images in terms of contrast, background, noise level and extraneous features. The micrographs also vary from sample to sample as a result of structural differences between different muscles and different species. The problem is to locate the individual filaments within a myofibril and to determine their orientations. Inspection of Fig. 2 shows that the filament boundaries are indistinct and their shapes not clear, making this a difficult task. Our overall approach is to first locate the filaments by filtering the

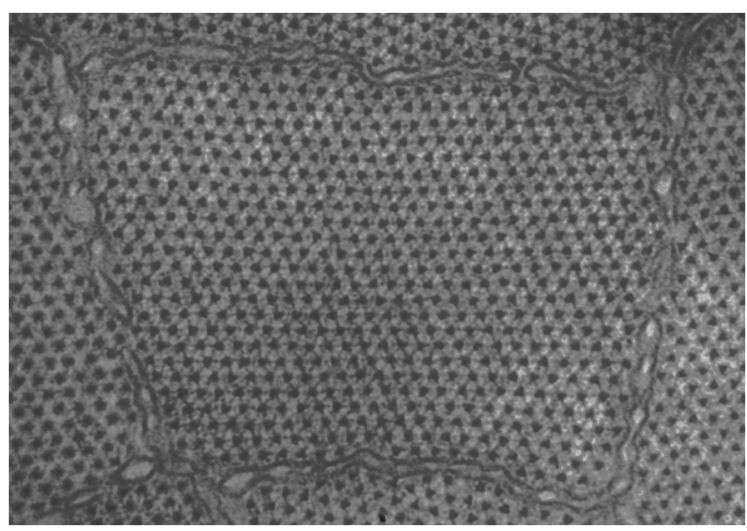

Fig. 2. Electron micrograph of a section through the bare region of frog sartorius muscle.

image with a point spread function that incorporates the local hexagonal symmetry. The orientation of each filament is then determined by template matching with a template that contains the salient features of the filament cross sections. The orientations are then classified using a Gaussian mixture model. The algorithm is assessed by comparison of the results with those obtained from manual analysis.

Location of the myosin filaments in the images is described in Section II. Determination of the individual filament orientations is described in Section III, and classification of the orientations is described in Section IV. Results of application of the method to a variety of micrographs and comparison to manual analyses are presented in Section V. Concluding remarks are made in Section VI.

\section{LOCATION OF THE FILAMENTS}

In order to determine the filament orientations, it is first necessary to locate the filaments in a micrograph. We have previously described an algorithm that uses grayscale morphology to determine the filament locations [11], [14]. This algorithm uses $h$-dome extraction [15] coupled with a neighbor analysis and use of lattice symmetry to optimize the threshold value, followed by further processing to correct erroneous locations. Although this algorithm is effective, it is quite complex and does not use the lattice symmetry in the most efficient manner.

We describe here a simpler approach to locating the filaments that uses matched filtering [16], [17] with a point spread function (psf) that incorporates the filament size and lattice symmetry at the outset. The lattice on which the filaments lie is very regular on a local scale, and the variation in the lattice is rather small over an entire myofibril. Furthermore, the filament diameter is approximately half the lattice spacing. We, therefore, use the local lattice symmetry to generate a psf that can be used to filter the whole micrograph image.

The psf is generated as follows. A reasonably clean area of the micrograph is chosen and the approximate coordinates of the center of one filament and its six nearest neighbors are manually selected. This is not difficult in practice and the approximate center coordinates do not need to be particularly accurate. This is the only manual step and effectively initializes the algorithm. The twelve nearest neighbor spacings between the filaments are 


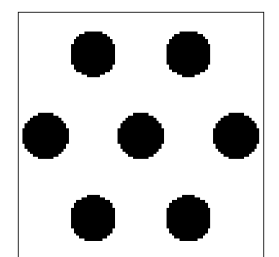

Fig. 3. Point spread function used to estimate the filament positions.

calculated and averaged to give the mean lattice spacing denoted $d$. (Note that this is different to the traditional "d-spacing" used in crystallography that refers to the perpendicular distance between the lattice planes). The orientations between the twelve nearest neighbor vectors are calculated and averaged modulo $60^{\circ}$. The average spacing and orientation is used to define a psf consisting of seven disks, each of diameter $d / 2$, arranged in a hexagon as shown in Fig. 3. The resulting binary image is then the psf for the given micrograph.

The psf is convolved with the image (implemented by multiplying the discrete Fourier transforms (DFT) of the image and the psf, and then calculating the inverse DFT), which produces a filtered image with distinct intensity peaks at the filament positions and a considerably reduced noise level compared to the original image. The advantage of this approach over the morphological approach is that the constraints of local maxima and local hexagonal symmetry are incorporated into a single step. The result is that a cleaner estimate of the peaks corresponding to the filaments is obtained more simply. The binary regional maxima image is then computed. The regional maxima are connected pixels (based on an 8-neighborhood) such that all the boundary pixels have equal value and connected pixels external to the region have strictly smaller values [15]. The centroids of the regional maxima structures are then taken as the estimates of the filament positions.

Application of the algorithm to a number of micrographs showed that it was effective in most cases. However, in particularly noisy micrographs some false positives were detected. Most false positives are eliminated by running the algorithm a second time using a psf with a spacing of $d+1$ pixels, and retaining only those filaments that are within 2 pixels of those initially determined. The algorithm then locates almost all of the filaments with sufficient accuracy for subsequent processing in all micrographs studied. An evaluation of the precision of the filament location algorithm is presented in Section V.

\section{DETERMINATION OF FILAMENT ORIENTATIONS}

Having determined the approximate locations of the filaments as described in the previous section, the next step is to estimate the orientation of each filament. This is achieved using template matching [18], [19]. The myosin filament cross sections have three-fold rotational symmetry and an approximate equilateral triangular shape. Also, the filaments do not have sharp outer edges, and the interior of the filaments is variable with some filaments having holes in their centers. We, therefore, use a template consisting of a hollow equilateral triangle with soft outer edges (described in more detail below).

The orientation of each filament is estimated by finding the orientation of the template that gives the largest correlation with

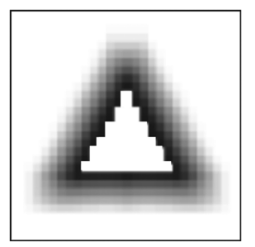

Fig. 4. Example of the template used to estimate the filament orientations.

the image of the filament. The most accurate estimate of the filament orientation is obtained if the template size and position accurately correspond to the size and position of the filament in the image. Since the filament sizes and positions are known only approximately, the best orientation is obtained by conducting a search over, in addition to the orientation $\theta$ of the template, the size $s$ (defined as the edge length of the triangle) and the position $(x, y)$ relative to the initial filament positions determined as described above. Note that since the optimum template is not spatially invariant, matched filtering cannot be used in this case. To allow fast calculation of the correlations, a family of templates of various sizes and orientations is precomputed and stored for later use. The range of sizes used is $d / 4<s<3 d / 4$ with $\triangle s=0.5$ pixels. As a result of the triangular shape, the unique set of orientations belongs to the interval $0<\theta<120^{\circ}$ and typically $\triangle \theta=4^{\circ}$ is used. Each template is constructed as an equilateral triangle of side length $s$ and with the values of the boundary pixels being proportional to the fraction of the pixel within the triangle. This is blurred with a square of $5 \times 5$ pixels, an interior triangle of edge length $s-2$ pixels is set to zero, and it is then rotated by the set of angles $\theta$. An example template is shown in Fig. 4.

Each filament in the micrograph image is correlated with each template for each position in the range $-3<x, y<3$ pixels in steps of 0.5 pixels, and the correlation coefficient

$$
r(x, y, s, \theta)=\frac{1}{n-1} \sum_{i=1}^{n}\left[\frac{\left[t(x, y, s, \theta, i)-\mu_{t}\right]\left[f(i)-\mu_{f}\right]}{\sigma_{t} \sigma_{f}}\right]
$$

calculated. Note that use of the correlation function ensures appropriate normalization [18], [19]. In (1), $t(x, y, s, \theta, i)$ and $f(i)$ are the template and filament values, respectively, at pixel $i, \mu$ and $\sigma$ are the mean and standard deviation respectively, and the sum is over the $n$ pixels that overlap in the filament image and the template. The estimate of each filament orientation, $\theta$, is that which maximizes the correlation, i.e.,

$$
\theta=\arg \max _{\theta^{\prime}}\left[\max _{x, y, s} r\left(x, y, s, \theta^{\prime}\right)\right] .
$$

For each filament, the larger the maximum correlation, the more reliable is the estimate of the orientation. Filaments that are particularly noisy or are less triangular in shape give smaller correlation coefficients and, therefore, less reliable orientations. The mean correlation coefficient over all the filaments, denoted $\bar{r}$, is calculated and is used as a measure of the overall quality of the image in terms of determining the filament orientations. Filaments that give correlation coefficients that are less than a chosen threshold value, denoted $r_{\text {min }}$, are considered to have estimated orientations that are too unreliable and their orientations 
are, therefore, marked as being unknown. A suitable threshold value varies from image to image although a value of 0.75 was found to be suitable in many cases.

\section{ClASSIFICATION OF ORIENTATIONS}

As described in the introduction, the myosin filaments in the micrographs with which we are concerned each adopt one of two orientations that are approximately $60^{\circ}$ apart, and it is the spatial distribution of these two orientations that is of interest. The objective is to classify each filament into one of two populations which represent the two sets of orientations. We refer to these two sets of orientations as up and down. Since the filament orientations have been defined to belong to $\theta \in\left(0^{\circ}, 120^{\circ}\right)$, the difference in orientations of "oppositely oriented" filaments is approximately $\pm 60^{\circ}$. As a result of imperfections in the muscle and errors in determining the orientations, the measured filament orientations do not belong to two groups that are exactly $60^{\circ}$ apart, but fall into two narrow distributions whose means are $\sim 60^{\circ}$ apart. The underlying distribution of orientations is modeled as a Gaussian mixture consisting of two normal distributions, and the classification is achieved by fitting the measured orientation histogram to this model. Since the orientations exist on the finite interval $\left(0^{\circ}, 120^{\circ}\right)$, each normal distribution for orientation $\theta$ is wrapped on this interval and denoted by $f(\mu, \sigma, \theta)$, where $\mu$ and $\sigma$ are the mean and standard deviation of each original normal distribution. The model density of the filament orientations $f_{\text {model }}(\theta)$ is then given by

$f_{\text {model }}(\theta)=p_{\text {up }} f\left(\mu_{\text {up }}, \sigma_{\text {up }}, \theta\right)+\left(1-p_{\text {up }}\right) f\left(\mu_{\text {down }}, \sigma_{\text {down }}, \theta\right)$

where $p_{\text {up }}$ is the proportion of up filaments. The parameters $\mu_{\text {up }}$, $\mu_{\text {down }}, \sigma_{\text {up }}, \sigma_{\text {down }}$, and $p_{\text {up }}$ are determined by fitting $f_{\text {model }}\left(\theta_{i}\right)$ to the histogram of estimated filament orientations $h\left(\theta_{i}\right)$ over the whole image by minimizing the sum of squared errors (SSE) over these parameters, where

$$
\begin{aligned}
S S E\left(\mu_{\mathrm{up}}, \mu_{\mathrm{down}}, \sigma_{\mathrm{up}}, \sigma_{\mathrm{down}}, p_{\mathrm{up}}\right) \\
=\sum_{\theta_{i}}\left[N f_{\text {model }}\left(\theta_{i}\right)-h\left(\theta_{i}\right)\right]^{2}
\end{aligned}
$$

and $N$ is the number of measured filament orientations. The minimization is performed using the Matlab function fminsearch. Ideally, the two populations will be separated by $60^{\circ}$, there will be a small spread of the orientations within each population, and the number of filaments in each population will be approximately equal. We, therefore, expect that $\Delta \mu=\left|\mu_{\text {up }}-\mu_{\text {down }}\right| \approx 60^{\circ}, \sigma_{\text {up }} \ll 60^{\circ}, \sigma_{\text {down }} \ll 60^{\circ}$ and $p_{\text {up }} \approx 0.5$. If these conditions are not satisfied then the orientation data are not consistent with the kind of distribution we expect. This could be due to a noisy image in which the filament orientations are not accurately enough determined, or to the filament orientations not belonging to two populations. The latter case may occur if the lattice contains dislocations so that different pairs of orientations occur in different regions of the image, or if the specimen contains only one filament orientation (as occurs in some kinds of muscle). The quantities $\Delta \mu$, $\sigma_{\text {up }}, \sigma_{\text {down }}$, and $p_{\text {up }}$ are, therefore, used to assess the quality

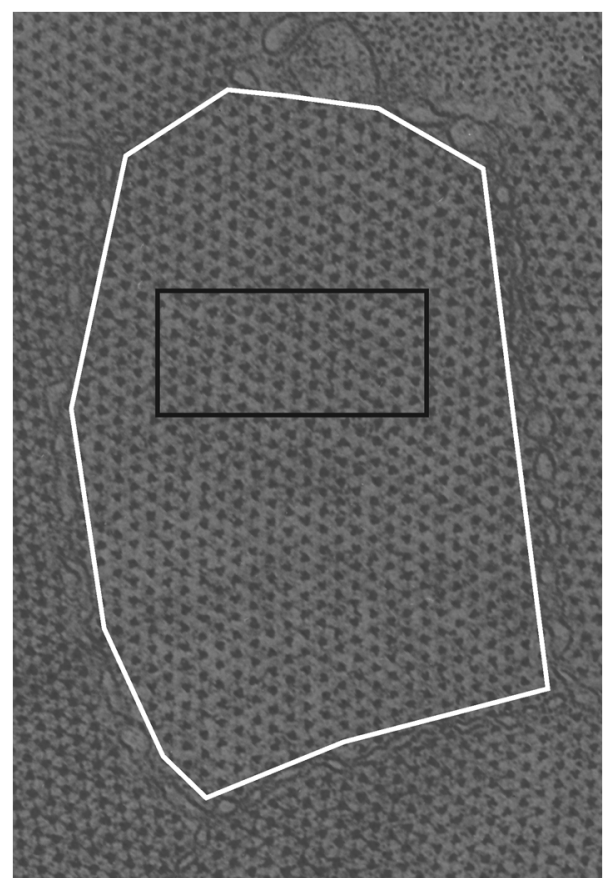

Fig. 5. Part of an electron micrograph of frog sartorius muscle [5]. The white border shows the region of one myofibril. The black border denotes the subimage shown in Figs. 6 and 8 .

of the micrograph and of the analysis. After fitting the model distribution, each filament is assigned to either population using

$$
\theta_{i} \in \begin{cases}\pi_{\mathrm{up}}, & \text { if } p_{\text {up }} f\left(\mu_{\mathrm{up}}, \sigma_{\mathrm{up}}, \theta_{i}\right) \\ & \geq p_{\text {down }} f\left(\mu_{\text {down }}, \sigma_{\text {down }}, \theta_{i}\right) \\ \pi_{\text {down }}, & \text { otherwise }\end{cases}
$$

where $\pi_{\text {up }}$ and $\pi_{\text {down }}$ represent two populations. This classification minimizes the expected number of misclassifications. With each filament orientation assigned to one of the two populations, the analysis is complete. Of course the filaments whose orientations are not determined as described in the previous section are not classified.

\section{Applications}

The algorithms described above were implemented in Matlab (MathWorks, Natick, MA) and run on a 2.8-GHz Pentium 4 PC with 1-GB of RAM. Results are presented here for applications to three superlattice muscle micrographs. Results are also presented for application to a simple lattice micrograph. Dissected muscle fibers were fixed, dehydrated and embedded in Araldite, and thin transverse sections cut with an ultramicrotome and stained [5]. Micrographs were obtained in a JEOL $1200 \mathrm{CX}$ electron microscope at a nominal $20,000 \times$ magnification. Images were digitized at 8 bits with a $20 \mu \mathrm{m}$ raster spacing on a Leafscan 45 film scanner.

To assess the precision of the algorithm, the automatic classification of the orientations was compared with a classification based on manually determined orientations. For the manual analysis, a program was used which allows the user to visually fit an equilateral triangle with adjustable position, angle and size to each filament displayed on a computer monitor. The manually determined orientations are then classified automatically as 


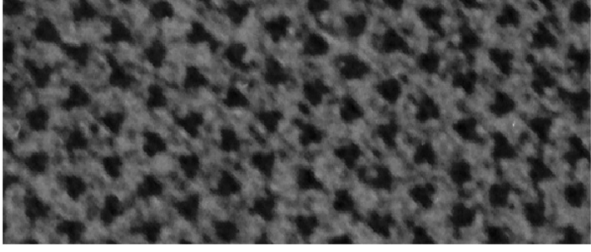

(a)

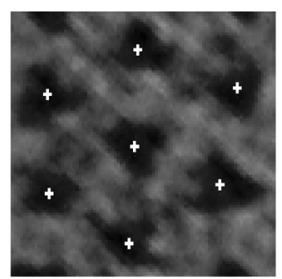

(b)

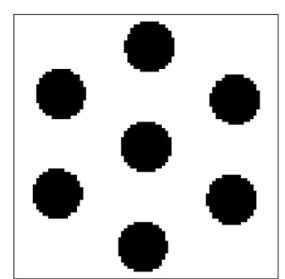

(c)

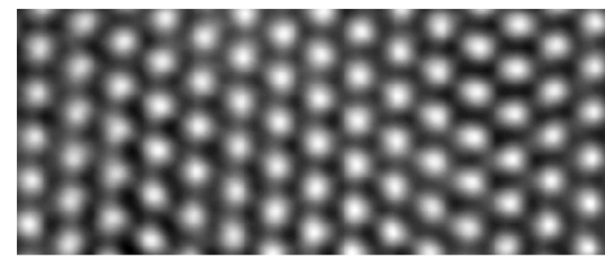

(d)

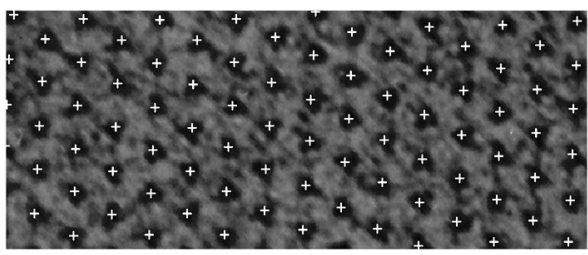

(e)

Fig. 6. Illustration of location of the filaments in the subimage shown in Fig. 5. (a) The original subimage, (b) the manually located filament positions used to determine the psf, (c) the psf, (d) the filtered subimage, and (e) the estimated filament positions.

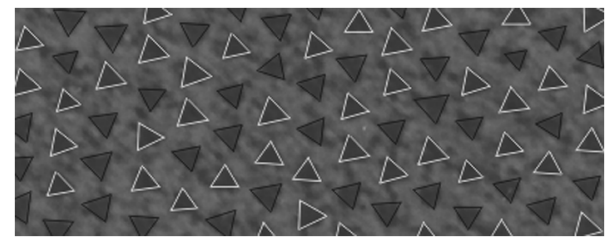

Fig. 7. Templates fitted to the filaments in the frog sartorius muscle subimage. The white and black triangles denote the classification into up and down orientations.

described above. Orientations that cannot be reliably estimated manually are marked as unknown and are not used in the classification.

The first micrograph is of a cross section of frog sartorius muscle [5] and the region containing one myofibril (inside the white border) is shown in Fig. 5. The raster spacing for this image is about $1 \mathrm{~nm} /$ pixel. A careful, although tedious, manual analysis of this image was performed and 820 filaments were located in the myofibril. The filament location algorithm described in Section II was run and 818 filaments were located. Relative to the manual analysis, the automatic analysis gave three false

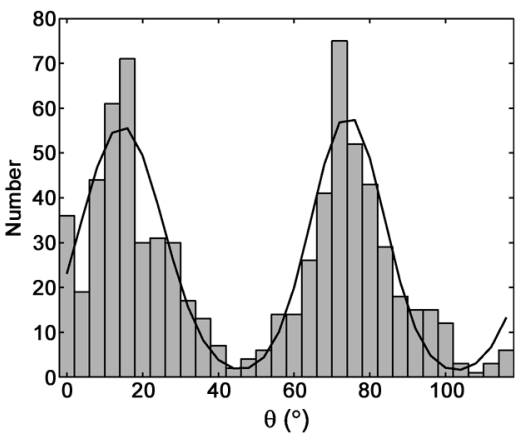

Fig. 8. Histogram and the fitted mixture model for the frog sartorius muscle micrograph.

TABLE I

PARAMETERS FOR THE DETERMINATION AND ANALYSIS OF MYOSIN FILAMENT ORIENTATIONS FOR THE VARIOUS MICROGRAPHS ANALYSED

\begin{tabular}{|c|c|c|c|c|c|}
\hline \multirow{2}{*}{ Parameter } & \multicolumn{5}{|c|}{ Image } \\
\cline { 2 - 6 } & Frog & Turtle & Polypterus & $\begin{array}{c}\text { Polypterus } \\
\text { Region A }\end{array}$ & $\begin{array}{c}\text { Polypterus } \\
\text { Region B }\end{array}$ \\
\hline $\bar{r}$ & 0.80 & 0.80 & 0.81 & 0.81 & 0.81 \\
$\Delta \mu\left(^{\circ}\right)$ & 60 & 62 & 55 & 61 & 58 \\
$\sigma_{\text {up }}\left({ }^{\circ}\right)$ & 11 & 15 & 35 & 7 & 12 \\
$\sigma_{\text {down }}\left(^{\circ}\right)$ & 10 & 15 & 10 & 15 & 13 \\
$p_{\text {up }}$ & 0.52 & 0.54 & 0.84 & 0.44 & 0.50 \\
\hline
\end{tabular}

TABLE II

COMPARISON OF THE AUtOMATIC AND MANUALly DETERMined ORIENTATIONS FROM THE FROG SARTORIUS MUSCLE MicROGRAPH

\begin{tabular}{|c|c|c|c|c|c|}
\hline \multirow{2}{*}{ Classification } & \multicolumn{4}{|c|}{ Automatic Orientations } \\
\cline { 3 - 6 } \multicolumn{2}{|c|}{} & Up & Down & Unknown & Total \\
\hline \multirow{3}{*}{ Manual } & Up & 335 & 9 & 25 & 369 \\
Orientations & Down & 7 & 318 & 22 & 347 \\
& Unknown & 32 & 37 & 30 & 99 \\
& Total & 374 & 364 & 77 & 815 \\
\hline
\end{tabular}

positives and five false negatives. The false alarm rates of $0.4 \%$ and $0.6 \%$ are considered quite low. The elapsed time for the processing was less than one minute. The operation of the location algorithm is illustrated in Fig. 6 which shows a subimage (corresponding to the region outlined in black in Fig. 5), together with the region used to define the psf, the psf, the filtered subimage, the regional maxima image, and the final filament positions. The mean distance between the automatically and manually determined filament positions was 1.4 pixels which corresponds to $1.4 \mathrm{~nm}$. This is small compared to the filament dimensions $(15 \mathrm{~nm})$ and is within the precision needed to determine the filament orientations.

The filament orientations were determined automatically as described in Section III and took two minutes. The mean correlation coefficient for the orientation of all filaments was $\bar{r}=$ 0.80 , and $738(90 \%)$ of the filaments have reliable orientations $\left(r>r_{\min }=0.75\right)$. The fitting of the templates is illustrated for the subimage in Fig. 7. The histogram of orientations is shown in Fig. 8 and two populations are evident. Fitting of 


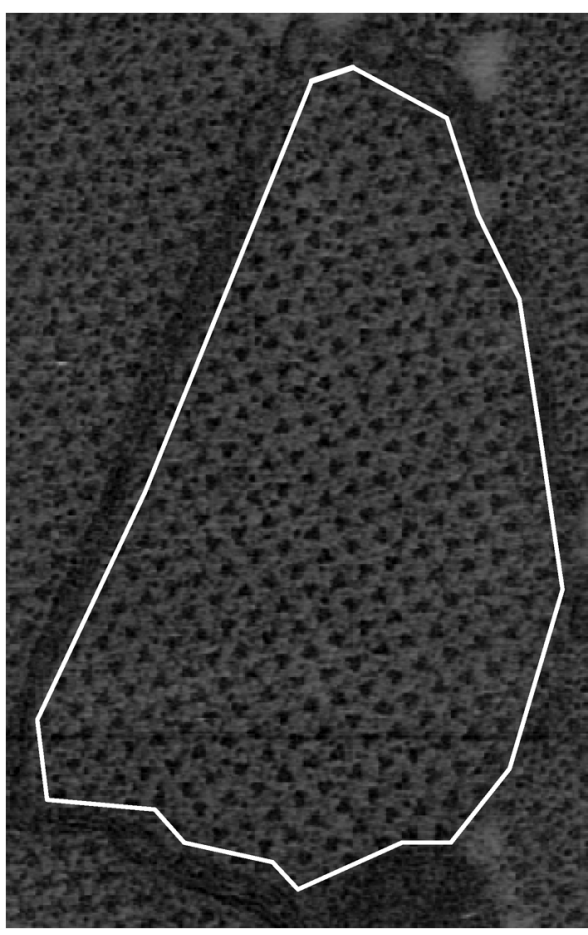

Fig. 9. Electron micrograph of a turtle muscle cross section.

the mixture model to the histogram gives good agreement as shown in Fig. 8. The five parameters described above are listed in Table I and their values indicate good quality data that are consistent with two populations of filament orientations. On the basis of the mixture model, the orientations were classified into two populations as described above, and the classifications for the subimage are illustrated in Fig. 7.

The filament orientations were also determined manually and classified automatically as described above. A careful manual analysis was able to determine 720 (88\%) filament orientations, slightly fewer than those determined automatically. A comparison of the results of the automatic and manual classifications is shown in Table II. The table shows the number of filaments classified as indicated by each of the two, automatic and manual, methods. As seen in the table, the automatically determined orientations are consistent overall with the manual determinations. Of the 738 automatically determined orientations, $653(88 \%)$ agree with the manually determined orientations, only $16(2 \%)$ have the opposite orientation, and the remaining $69(10 \%)$ could not be determined manually. Overall, the automatic classification is consistent with that based on a manual determination of filament orientations, keeping in mind that the manual classification cannot be considered "correct."

The second micrograph analysed is from turtle leg muscle and is shown in Fig. 9. The raster spacing is about $1.5 \mathrm{~nm} /$ pixel. This image is noisier than that from the frog sartorius muscle (Fig. 5). The myosin filaments do not have as clearly defined triangular profiles and the image contains many small features (possibly parts of the actin filaments) between the myosin filaments, making both manual and automated analysis more difficult. The noisy nature of the image is more apparent in the subimage shown in Fig. 10(a). The filament location algorithm located 332 filaments with only 1 false positive and 6 false nega-

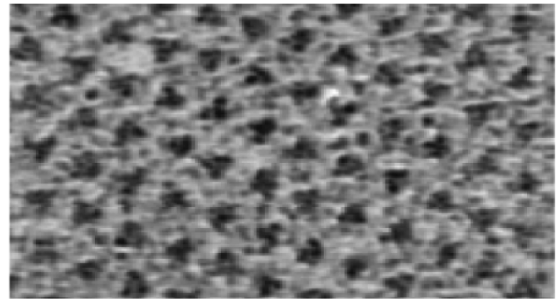

(a)

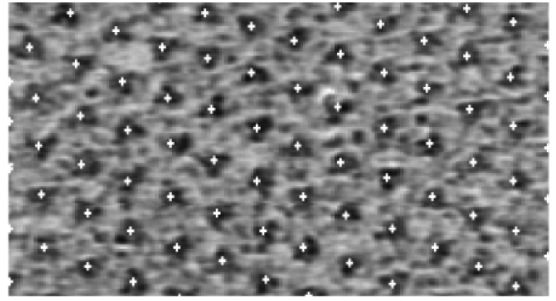

(b)

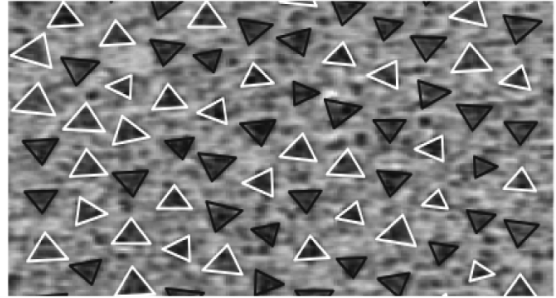

(c)

Fig. 10. (a) Subimage of the turtle muscle micrograph, (b) the determined filament locations, and (c) the classification of the filament orientations.

TABLE III

COMPARISON OF THE AUTOMATIC AND MANUALLY DETERMINED ORIENTATIONS FROM THE TURTLE LEG MUSCLE MiCROGRAPH

\begin{tabular}{|c|c|c|c|c|c|}
\hline \multirow{2}{*}{ Classification } & \multicolumn{4}{c|}{ Automatic Orientations } \\
\cline { 3 - 6 } & Up & Down & Unknown & Total \\
\hline \multirow{3}{*}{ Manual } & Up & 96 & 7 & 12 & 115 \\
Orientations & Down & 12 & 104 & 18 & 134 \\
& Unknown & 21 & 40 & 22 & 83 \\
& Total & 129 & 151 & 52 & 332 \\
\hline
\end{tabular}

tives relative to a manual analysis. The filament location is illustrated for the subimage in Fig. 10(b). The filament orientations were determined automatically and the mean correlation coefficient was 0.80 . Using a correlation threshold of $0.75,280$ of the filament orientations $(84 \%)$ were reliably determined. Fitting the Gaussian mixture model gave the parameters listed in Table I, indicating a good classification. The results of the classification for the subimage are shown in Fig. 10(c). Manual analysis gave the orientations of 249 (75\%) filaments, which were then classified automatically. A comparison of the manual and automatic classifications are shown in Table III. Of the automatically determined orientations, $200(71 \%)$ agree with the manually determined classifications and $19(7 \%)$ have the opposite classification. The proportion of opposite manually and automatically determined orientations is larger than in the first example as a result of the higher level of noise in this image. However, comparison of two manual analyses of this micrograph by 


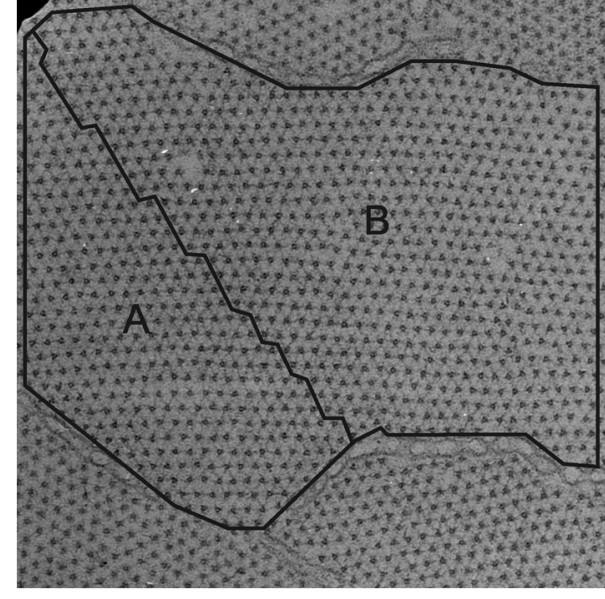

Fig. 11. Electron micrograph of a Polypterus muscle cross section. The border between the two regions $\mathrm{A}$ and $\mathrm{B}$ is shown.

two operators results in a similar percentage of filaments being labelled with opposite classifications, indicating that the automatic method is at least as good as a manual analysis. In summary, the automatic procedure classified more filament orientations than did the manual analysis, and the results of the two analyses are consistent overall, indicating good performance of the algorithm.

The third micrograph is from Polypterus fish muscle and is shown in Fig. 11. Polypterus is a ray-finned fish that falls outside the group of classical teleost fish which have the simple lattice structure. The raster spacing is about $1.5 \mathrm{~nm} /$ pixel. Automatic analysis of this micrograph located 1044 filaments and the orientations of 986 (94\%) of these were reliably determined using a correlation threshold of 0.75 . The histogram of orientations is shown in Fig. 12(a), and fitting the mixture model gave the density shown in Fig. 12(a) and the parameters listed in Table I. Inspection of the histogram, however, shows no evidence of two populations of orientations as expected. This is supported by the values of some of the parameters listed in Table I, particularly the large standard deviation of one of the Gaussian components. The filament orientations in this micrograph do not evidently fall into two populations. However, a careful inspection shows evidence of two differently orientated pairs of orientations, each concentrated in two regions of the micrograph. Although there is no distinct interface between these two regions, a border was estimated and the image partitioned into two regions labelled A and B as shown in Fig. 11. The regions A and B contain 317 and 663 reliably determined filament orientations, respectively. The filament orientations in each region were classified separately. The resulting histograms and the fitted densities are shown in Fig. 12(b) and (c) and the resulting parameters are listed in Table I. Both the histograms and the values of the parameters indicate good classifications in each region. However, the pairs of orientations in the two regions are rotated by $\sim 30^{\circ}$ relative to each other. Evidently then, the myofibril is segregated into two regions with a high degree of orientational order within each region. In previous studies, some micrographs of sections not exactly transverse to the myofibril that cross the M-band show a rotation of $\sim 30^{\circ}$ in the filament orientations between the bare regions on either side of the M-band [20]. The

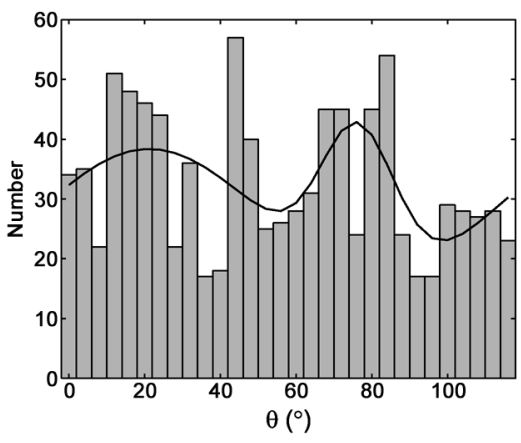

(a)

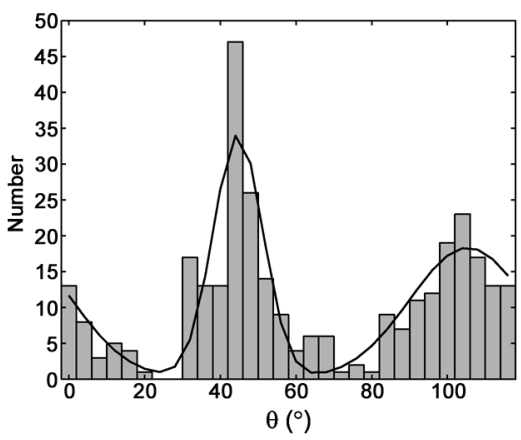

(b)

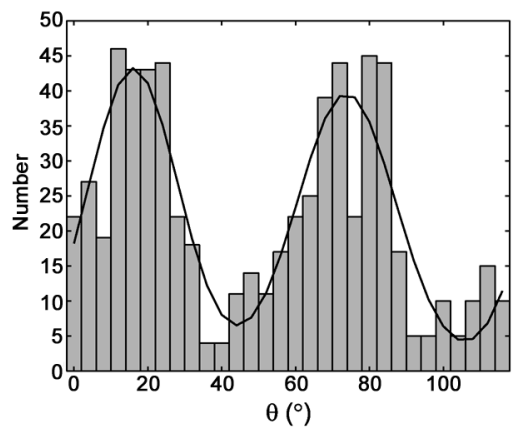

(c)

Fig. 12. Histograms and mixture models for the Polypterus muscle lattice for (a) the whole micrograph, (b) the region $\mathrm{A}$, and (c) the region $\mathrm{B}$.

myofibril in Fig. 11 does not show a clear central M-band region where the filament profiles are nearly circular [20], and so the change in rotation is probably due to a dislocation in the lattice.

The fourth micrograph is from mudskipper fish muscle and is shown in Fig. 13(a). The raster spacing is about $1.3 \mathrm{~nm} /$ pixel. Automatic analysis of this micrograph located 171 filaments and the orientations of $156(91 \%)$ of these were reliably determined using a correlation threshold of 0.70 . Fitting the Gaussian mixture model to the orientations gave $\Delta \mu=32^{\circ}$ and $p_{\text {up }}=$ 0.92 , indicating a poor fit to the model. However, fitting a single Gaussian gave a good fit with $\sigma=11^{\circ}$ as shown in Fig. 13(b). The myosin filaments of mudskipper fish muscle are known to form a simple lattice, i.e. they adopt a single orientation. Our algorithm, therefore, also satisfactorily detects the presence of the simple lattice.

\section{CONCLUSION}

Electron micrographs of cross sections of the bare region of vertebrate muscle show the myosin filaments in a noisy and vari- 


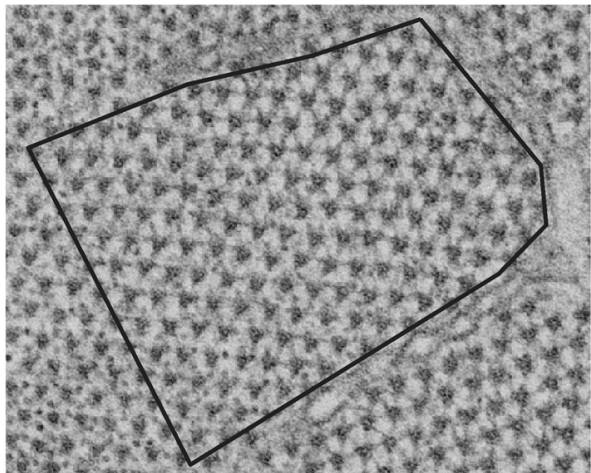

(a)

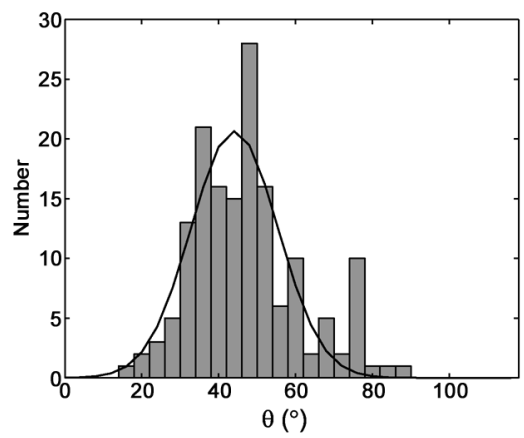

(b)

Fig. 13. (a) Subimage of the mudskipper muscle cross section, and (b) its histogram and Gaussian model.

able background. Determination and classification of the filament rotations is an important step in analysing the disorder in the myosin lattice, which is of relevance for muscle structure and function as well as for analysis of X-ray fiber diffraction data from muscle. Currently used manual methods for analysis of filament orientations in such images are unsatisfactory in terms of accuracy, reproducibility and the time they require. The automatic algorithm described allows effective and rapid analysis of micrographs to determine the spatial distribution of filament orientations. The algorithm was applied successfully to a number of micrographs of various qualities for which manual analysis was possible. Comparison of the results obtained with those of manual analyses shows a good consistency, indicating that the automatic analysis is at least as accurate as, and probably more accurate than, manual analysis.

\section{REFERENCES}

[1] J. M. Squire, The Structural Basis of Muscular Contraction. London, U.K.: Plenum, 1981.

[2] J. M. Squire, "Architecture and function in the muscle sarcomere," Curr. Opin. Struct. Biol., vol. 7, pp. 247-257, 1997.

[3] J. M. Squire, P. K. Luther, and P. Morris, "Organisation and properties of the striated muscle sarcomere," in Molecular Mechanisms in Muscular Contraction, J. M. Squire, Ed. Boca Raton, FL: CRC, 1990, pp. $1-48$.

[4] M. A. Geeves and K. C. Holmes, "The molecular mechanism of muscular contraction," Adv. Protein Chem., vol. 71, pp. 161-193, 2005.

[5] P. K. Luther and J. M. Squire, "Three-dimensional structure of the vertebrate muscle A-band II. The myosin filament superlattice," J. Mol. Biol, vol. 141, pp. 409-439, 1980.
[6] P. K. Luther, J. M. Squire, and P. L. Forey, "Evolution of myosin filament arrangements in vertebrate skeletal muscle," J. Morphol., vol. 229, pp. 325-335, 1996.

[7] C. R. Bagshaw, Muscle Contraction. London, U.K.: Chapman \& Hall, 1982.

[8] H. E. Huxley and W. Brown, "The low-angle X-ray diagram of vertebrate striated muscle and its behaviour during contraction and rigor," J. Mol. Biol., vol. 30, pp. 383-434, 1967.

[9] G. Offer, "The molecular basis of muscular contraction," in Companion to Biochemistry, A. T. Bull, J. R. Lagnado, J. O. Thomas, and K. F. Tipton, Eds. London, U.K.: Longman, 1974, pp. 623-671.

[10] R. P. Millane and A. Goyal, "Analysis of the disordered myosin lattice in muscle," Fibre Diffraction Rev., vol. 4, pp. 6-11, 2000.

[11] B. Bödvarsson, S. Klim, S. Mortensen, M. Mørkebjerg, J. Chen, J. R. Maclaren, C. H. Yoon, P. K. Luther, J. M. Squire, A. Bainbridge-Smith, P. J. Bones, and R. P. Millane, "Determination of myosin filament positions and orientations in electron micrographs of muscle cross-sections," in Proc. SPIE Image Reconstruction From Incomplete Data III, 2004, vol. 5562, pp. 97-108.

[12] C. H. Yoon, J. Chen, J. R. Maclaren, B. Bödvarsson, S. Klim, S. Mortensen, M. Mørkebjerg, P. K. Luther, J. M. Squire, A. Bainbridge-Smith, P. J. Bones, and R. P. Millane, "Automated analysis of electron micrographs of muscle cross-sections," in Proc. Image and Vision Computing New Zealand, D. Pairman, H. North, and S. McNeill, Eds., NZ, 2004, pp. 173-178, Landcare Research.

[13] C. H. Yoon, P. J. Bones, and R. P. Millane, "Image analysis for electron microscopy of muscle fibres," in Proc. Digital Image Computing: Techniques and Applications, B. C. Lovell, A. J. Maeder, S. Ourselin, and T. Caelli, Eds., 2005, pp. 79/1-79/7, IEEE CS Press.

[14] B. Bödvarsson, S. Klim, S. Mortensen, M. Mørkebjerg, C. H. Yoon, J. Chen, J. R. Maclaren, P. K. Luther, J. M. Squire, P. J. Bones, and R. P. Millane, "A morphological image processing method for locating myosin filaments in muscle electron micrographs," Image Vis. Comput., vol. 26, pp. 1073-1080, 2008.

[15] L. Vincent, "Morphological grayscale reconstruction in image analysis: Applications and efficient algorithms," IEEE Trans. Image Process., vol. 2, pp. 176-201, Apr. 1993.

[16] A. Rosenfeld and C. Kak, Digital Picture Processing, 2nd ed. New York: Academic, 1982, vol. 2.

[17] A. K. Jain, Fundamentals of Digital Image Processing. Englewood Cliffs, NJ: Prentice-Hall, 1989.

[18] J. C. Russ, The Image Processing Handbook, 4th ed. Boca Raton, FL: CRC, 2002.

[19] M. S. Nixon and A. S. Aguado, Feature Extraction and Image Processing. Oxford, U.K.: Newnes, 2002.

[20] P. K. Luther, P. M. G. Munro, and J. M. Squire, "Three-dimensional structure of the vertebrate muscle A-band. III. M-region structure and myosin filament symmetry," J. Mol. Biol., vol. 151, pp. 703-730, 1981.

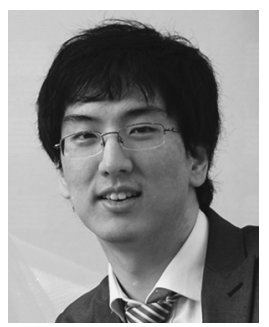

Chunhong H. Yoon received the B.E. (Hons.) degree in electrical and electronic engineering from the University of Canterbury, Christchurch, New Zealand, in 2003. He is currently pursuing the Ph.D. degree in the Department of Electrical and Computer Engineering, University of Canterbury.

His research involves modeling and analysis of disordered biological systems.

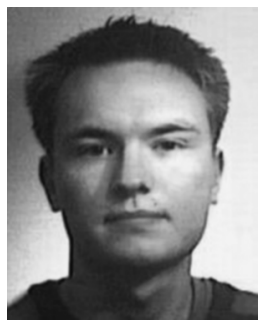

Bjarni Bödvarsson received the M.Sc.Eng. degree from the Technical University of Denmark (DTU) in 2006.

He was a visiting researcher in the Department of Electrical and Computer Engineering, University of Canterbury, Christchurch, New Zealand, in 2003. He worked as a Ph.D. student from 2006 to 2007 and is currently working as a System Development Consultant for SimCorp A/S which produces investment management software for the financial industry. 


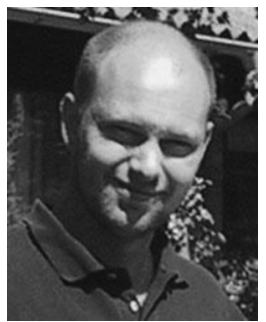

Søren Klim received the M.Sc. degree in engineering from the Technical University of Denmark (DTU) in 2006.

He was a visiting researcher in the Department of Electrical and Computer Engineering, University of Canterbury, Christchurch, New Zealand, in 2003. He is currently enrolled in an industrial Ph.D. program in collaboration with Novo Nordisk A/S and DTU working on insulin and glucose modeling.

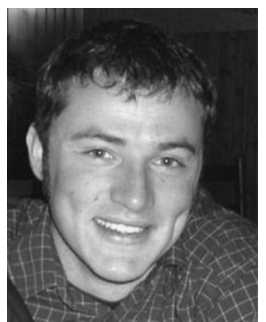

Martin Mørkebjerg received the M.Sc.Eng. degree from the Technical University of Denmark (DTU) in 2006.

He was a visiting researcher in the Department of Electrical and Computer Engineering at the University of Canterbury, Christchurch, New Zealand, in 2003. He is currently working as a Research Engineer at the R\&D Department of the hearing aid company Widex A/S.

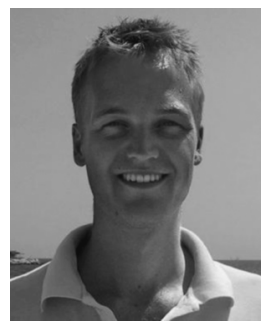

Stig Mortensen received the M.Sc.Eng. degree from the Technical University of Denmark (DTU) in 2006. He is currently pursuing the Ph.D. degree at DTU in the Department of Informatics and Mathematical Modelling on a project concerned with dynamic analysis of sleep EEG data in relation to pharmacokinetics.

He was a visiting researcher in the Department of Electrical and Computer Engineering at the University of Canterbury, Christchurch, New Zealand, in 2003.

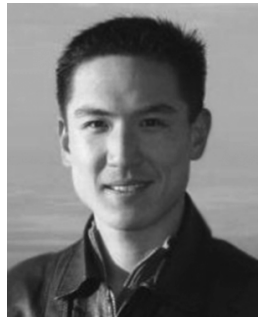

James Chen (S'01) received the B.E. (Hons.) degree in electrical and electronic engineering from the University of Canterbury, Christchurch, New Zealand, in 2004, and the M.S. degree in electrical engineering from Stanford University, Stanford, CA, in 2007. He is currently purusing the Ph.D. degree in the Department of Electrical Engineering, Stanford University, where his research involves the design of energy-efficient circuits for processors and on-chip networks.

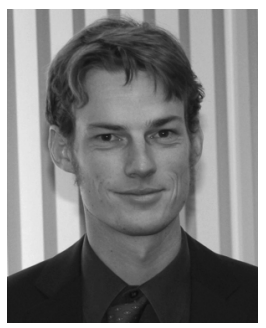

Julian R. Maclaren received the B.E. (Hons.) and $\mathrm{Ph} . \mathrm{D}$. degrees in electrical and electronic engineering from the University of Canterbury, Christchurch, New Zealand, in 2004 and 2008, respectively.

$\mathrm{He}$ is currently a Research Scientist in the Department of Diagnostic Radiology, University Hospital Freiburg, Freiburg, Germany, where he is working on motion correction in magnetic resonance imaging.

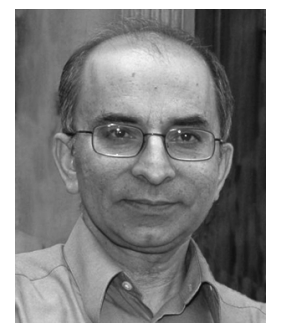

Pradeep K. Luther received the B.Sc. degree in physics and the Ph.D. degree in biophysics from Imperial College, London, U.K., in 1974 and 1978, respectively.

He has worked in the Physics Departments at the University of Nairobi, Kenya, and Imperial College, London. He is currently a Principal Research Fellow in the Faculty of Medicine at Imperial College where he studies muscle structure using electron microscopy, electron tomography and image processing.

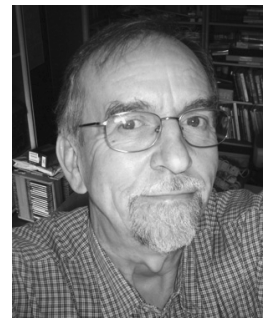

John M. Squire received the B.Sc. degree in physics and the Ph.D. degree in biophysics from King's College, London, U.K., in 1966 and 1969, respectively.

$\mathrm{He}$ is a Professor of structural biophysics in the University of London and was Head of the Biological Structure and Function Section at Imperial College London until he retired. He is currently an Honorary Research Fellow in the Department of Physiology and Pharmacology, University of Bristol, Bristol, U.K., and Visiting Professor at Imperial College London. He studies muscle structure and function using electron microscopy, X-ray fiber diffraction, time-resolved diffraction, and protein crystallography.

Prof. Squire is a Fellow of the Institute of Physics and the Institute of Biology and an Honorary Member of the British Biophysical Society.

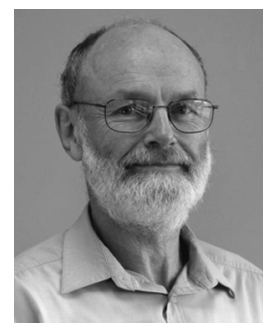

Philip J. Bones (M'81-SM'93) received the B.E. (Hons), M.E., and Ph.D. degrees in electrical engineering from the University of Canterbury, Christchurch, New Zealand, in 1973, 1975, and 1981 , respectively.

He worked for 11 years as a Biomedical Engineer in the Department of Cardiology, Christchurch Hospital, Christchurch, and for two years as Postdoctoral Fellow with cardiac groups in Europe (including one year as Alexander von Humboldt fellow in Heidelberg, Germany). Since 1988, he has been in the Department of Electrical and Computer Engineering at the University of Canterbury, Christchurch, New Zealand. His research interests include medical imaging, image recovery, and signal processing applied to physiological signals.

Dr. Bones is a member of the SPIE and ACPSEM (Australasian College of Physical Scientists and Engineers in Medicine).

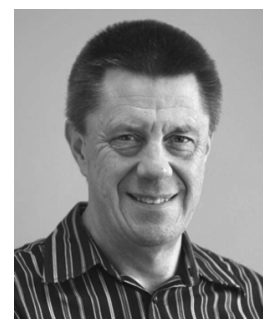

R. P. Millane (M'82-SM'94) received the B.E. (Hons.) and Ph.D. degrees in electrical engineering from the University of Canterbury, Christchurch, New Zealand, in 1975 and 1981, respectively.

He was on the faculty of Purdue University, Wes Lafayette, IN, for 20 years. He was a visiting scientist in the Marine Physical Laboratory, Scripps Institution of Oceanography, La Jolla, CA, in 1999. He is currently Professor and Head of the Department of Electrical and Computer Engineering at the University Canterbury, Christchurch, New Zealand. His research interests are concerned with computational imaging and diffraction, with applications in biophysical and medical imaging, and remote sensing.

Dr. Millane is a Fellow of the Optical Society of America and a member of the Editorial Board of Acta Crystallographica Section A. He has served as chairman of OSA Topical Meetings on Signal Recovery and Synthesis and SPIE Conferences on Image Reconstruction from Incomplete Data. 